\title{
NICE Guidelines Cannot Be Recommended for Imaging Studies in Children Younger Than 3 Years with Urinary Tract Infection
}

\author{
Marko Tapani Ristola ${ }^{1}$ Timo Hurme ${ }^{1}$ \\ ${ }^{1}$ Department of Pediatric Surgery, Turku University Hospital, Turku, \\ Varsinais-Suomi, Finland \\ Address for correspondence Marko Tapani Ristola, BM, Department of \\ Pediatric Surgery, Turku University Hospital, PO Box 52, 20521, Turku, \\ Eur J Pediatr Surg 2015;25:414-420. \\ Finland (e-mail: mtrist@utu.fi).
}

\begin{abstract}
Introduction We assessed the possible consequences of applying the National Institute of Health and Clinical Excellence (NICE) guidelines for imaging studies of children younger than 3 years with urinary tract infection (UTI) to clinical practice, in terms of altered diagnoses and treatment.

Material and Methods In a retrospective cohort of 672 patients with UTI, we evaluated indications for and results of renal and bladder ultrasonography, voiding cystourethrography (VCUG), dimercaptosuccinic acid scintigraphy, UTI recurrence, antimicrobial prophylaxis (AMP), antireflux procedures, and other urological procedures.

Results There were a total 125 patients with vesicoureteral reflux (VUR), of whom 59 patients $(47 \%)$ would have been missed, had the NICE guidelines being applied. These

Keywords

- urinary tract infections

- vesicoureteral reflux

- child

- NICE guidelines included 20 of the 64 patients (31\%) with dilating VUR and 13 of the 30 patients (43\%) who underwent antireflux procedures. A VCUG would have been avoided in 184 patients (74\%) with no VUR in VCUG.

Conclusion Based on the results in this cohort of 672 patients, we cannot recommend the use of the NICE guidelines for imaging studies in children younger than 3 years with UTI.
\end{abstract}

\section{Introduction}

Urinary tract infections (UTIs) are common in young children: among infants presenting with fever, the overall prevalence of UTI is $7.0 \%$. After the 1st year of life UTIs become less frequent. ${ }^{1}$ In children younger than 3 years, recurrent, febrile UTIs, especially in the presence of vesicoureteral reflux (VUR) has been thought to lead to renal scarring and to contribute to the development of renal failure and hypertension later in life. ${ }^{2-4}$ This has led to the extensive use of imaging studies in children with UTI, in an attempt to identify patients with abnormal urinary tract anatomy or VUR. For identifying VUR, voiding cystourethrography (VCUG) still remains the gold

received

March 18, 2014

accepted

May 22, 2014

published online

July 31,2014

standard. VCUG, while effective for identifying patients with VUR, is not without disadvantages. It requires catheterizing the child and exposes the child to high doses of radiation, in addition to being a costly procedure.

Several guidelines have been presented regarding appropriate imaging strategies aiming at directing imaging studies to those patients who may benefit most from treatment. One of these guidelines has been issued by the National Institute of Health and Clinical Excellence (NICE). ${ }^{5}$ Stringent following of the guidelines limits the use of VCUG significantly, even compared with other similar guidelines. ${ }^{6-10}$ The NICE guidelines also restrict the use of renal and bladder ultrasonography (RBUS), which for decades has been a part of first-line

(c) 2015 Georg Thieme Verlag KG Stuttgart · New York
DOI http://dx.doi.org/ 10.1055/s-0034-1384646. ISSN 0939-7248. 
investigations in any child with UTI. Some studies have been performed about the use of the NICE guidelines, ${ }^{11-16}$ but the studies have not been conclusive, and the results have differed considerably. The authors of this article published a preliminary analysis of this study in 2013 in which we stated that the NICE guidelines may be applicable to clinical use in boys younger than 6 months, but not in other groups. ${ }^{12}$

This study evaluates a larger cohort to assess the applicability of the NICE guidelines for imaging studies in children younger than 3 years with a UTI, as compared with routine procedures at our institution (University Hospital of Turku), where over half of the patients underwent VCUG to identify VUR during the period under evaluation.

\section{Material and Methods}

The patient records of 672 patients treated for UTI in the University Hospital of Turku between January 1, 2000 and December 31, 2009 were reviewed. There were 292 boys (211 younger than 6 months, 81 older than 6 months but younger than 3 years), and 380 girls ( 117 younger than 6 months, 263 older than 6 months but younger than 3 years). Patients were excluded from the final analysis for the following reasons: (1) previously diagnosed urological abnormalities (e.g., hydronephrosis in antenatal ultrasound), (2) not undergoing RBUS, (3) having the imaging studies performed in another health care district, and (4) moving to another health care district before imaging studies at our institution.

All UTI diagnoses were reassessed by one of the authors (M. T. R.). The diagnosis of UTI was considered certain if there was bacterial growth of $100,000 \mathrm{CFU} / \mathrm{mL}$ in two urinary bag specimens or clean catch urine samples, or if there was any growth of a single uropathogen in a suprapubic aspiration sample. A blood C-reactive protein level of $40 \mathrm{mg} / \mathrm{L}$ or more and a fever of $38^{\circ} \mathrm{C}$ were the criteria for pyelonephritis (PN). Signs of renal inflammation in RBUS were also considered confirmation of a diagnosis of PN.

Antimicrobial treatment followed the routine procedures of our department, and the antibiotic drug of choice was determined according to the antibiogram of the uropathogen in each case. If a patient was started on antimicrobial treatment before the antibiogram was available, the drug of choice for PN was intravenous cefuroxime and for cystitis either a single intramuscular injection of ceftriaxone followed by oral antimicrobials, or only oral antimicrobials. Most patients with PN received intravenous cefuroxime for 3 days followed by oral antimicrobials after discharge from the hospital for a total treatment period of 10 days. The most common oral antimicrobials were trimethoprim-sulfamethoxazole, amoxicillin clavulanic acid, and cephalexine.

Data regarding the indications for RBUS and VCUG were recorded, the premise being that whenever a study was to be considered according to the NICE guidelines, it was to be performed.

All of the patients in our cohort underwent RBUS. If VUR was suspected, a VCUG was scheduled. If renal parenchymal damage was suspected, a dimercaptosuccinic acid (DMSA) scintigraphy was occasionally scheduled. Where urinary tract obstruction was suspected, a mercaptoacetyltriglycine renal scan was performed at the discretion of the responsible physician.

All patients' VUR was graded according to the criteria determined by the International Reflux Study in Children, ${ }^{17}$ and the highest grade detected in all studies was recorded for this study. The common finding in nuclear VCUG (nVCUG), "VUR is reaching the level of the kidney" without reference to dilation, was determined as grade 2 . However, data regarding dilation in RBUS were combined to the nVCUG finding to determine the grade of VUR as accurately as possible. If the patient had bilateral VUR, the higher grade of the two recordings was used for classifications.

The NICE guidelines were used to determine the indications for RBUS and VCUG and to define recurrent UTI and atypical UTI.

The mean follow-up time was 8.5 years (range 3.9-13.9 years). Data were gathered regarding UTI recurrence, use of antimicrobial prophylaxis (AMP), antireflux procedures, and other urological procedures and additional imaging studies. Data were analyzed as to what the consequences would have been, had the NICE guidelines being applied to clinical use in this cohort, with regard to "missed" diagnoses or treatment.

Statistical comparisons between any two groups were performed using a Fischer exact test: a $p$ value of $<0.05$ was considered statistically significant.

\section{Results}

\section{Renal and Bladder Ultrasonography}

Altogether 356 out of the 672 (53\%) children with UTI had an indication for RBUS before undergoing imaging studies. The remaining 316 patients underwent RBUS without an indication. ( - Table 1). Sixteen of the latter patients had an indication for RBUS later on during follow-up, after another episode of UTI.

All 672 patients underwent RBUS, and the result was abnormal in 142 (21\%) of these, including 92 cases (14\%) of renal and/or ureteral dilation (-Table 1). Of the 316 patients who underwent RBUS without an indication, the result was abnormal in 45 (14\%), including 21 patients (6.6\%) with dilation in RBUS (-Table 2).

\section{Voiding Cystourethrography}

In total, 165 patients (25\%) had an indication for VCUG before undergoing one. A VCUG was performed in 129 of these patients. Total 243 patients underwent VCUG without an indication (-Table 1). Four of these patients fulfilled the indication criteria for VCUG later on during follow-up, after another episode of UTI. In the older age group, boys were more likely to have an indication for VCUG than girls $(p=0.0026)$. There was no statistically significant difference in fulfilling the indication criteria for VCUG between boys and girls in the younger age group $(p=0.1574)$. In both the gender groups, younger patients were more likely to fulfill the indication criteria for VCUG $(p=0.0002$ and $p<0.0001$, respectively). 
Table 1 Data on 672 children with UTI

\begin{tabular}{|c|c|c|c|c|c|}
\hline Age group & Boys $<6$ mo & Girls $<6$ mo & Boys $0.5-3$ y & Girls $0.5-3$ y & All patients \\
\hline Total number of patients & 211 & 117 & 81 & 263 & 672 \\
\hline Indication for US & 192 & 104 & 19 & 41 & 356 \\
\hline US performed & 211 & 117 & 81 & 263 & 672 \\
\hline Abnormal US & 59 & 29 & 15 & 39 & 142 \\
\hline Dilation & 43 & 21 & 7 & 21 & 92 \\
\hline Hydronephrosis & 17 & 7 & 2 & 4 & 30 \\
\hline Mild pelvic or ureteral dilation & 26 & 14 & 5 & 17 & 62 \\
\hline Renal scarring & 10 & 6 & 5 & 13 & 34 \\
\hline Abnormal kidney size & 12 & 9 & 4 & 9 & 34 \\
\hline Duplicated ureter & 6 & 9 & 0 & 6 & 21 \\
\hline Ureterocele & 2 & 1 & 0 & 0 & 3 \\
\hline Nephrocalcinosis & 2 & 1 & 0 & 0 & 3 \\
\hline Bladder cyst & 0 & 0 & 1 & 1 & 2 \\
\hline Paraureteral diverticulum & 0 & 0 & 1 & 0 & 1 \\
\hline Urethral valve & 1 & 0 & 0 & 0 & 1 \\
\hline Urachal cyst & 0 & 1 & 0 & 0 & 1 \\
\hline Laterally placed UVJ & 0 & 0 & 0 & 1 & 1 \\
\hline Renal calculus & 0 & 0 & 0 & 1 & 1 \\
\hline Indication for VCUG & 90 & 40 & 16 & 19 & 165 \\
\hline VCUG performed & 152 & 72 & 37 & 111 & 372 \\
\hline VUR on VCUG & 46 & 30 & 16 & 33 & 125 \\
\hline Low-grade VUR & 24 & 13 & 6 & 18 & 61 \\
\hline High-grade VUR & 22 & 17 & 10 & 15 & 64 \\
\hline AMP for VUR & 44 & 27 & 12 & 27 & 110 \\
\hline Surgery & 15 & 10 & 8 & 16 & 49 \\
\hline Endoscopic injection treatment ${ }^{\mathrm{a}}$ & 4 & 8 & 3 & 15 & 30 \\
\hline Ureteroneocystostomy & 1 & 3 & 1 & 0 & 5 \\
\hline Nephrectomy & 0 & 0 & 2 & 0 & 2 \\
\hline Pyeloplasty & 5 & 0 & 1 & 1 & 7 \\
\hline Excision of ureterocele & 2 & 1 & 0 & 0 & 3 \\
\hline Urethral valve ablation & 1 & 0 & 1 & 0 & 2 \\
\hline Circumcision & 1 & 0 & 0 & 0 & 1 \\
\hline Nephrostomy & 1 & 0 & 0 & 0 & 1 \\
\hline
\end{tabular}

Abbreviations: AMP, antimicrobial prophylaxis; US, ultrasonography; UTI, urinary tract infection; UVJ, ureterovesical junction; VCUG, voiding cystourethrography; VUR, vesicoureteral reflux.

${ }^{\mathrm{a}}$ Two patients underwent endoscopic injection treatment despite being diagnosed with only low-grade VUR.

A total of 137 patients underwent only VCUG, 177 patients only nVCUG, 57 patients both. In addition, one patient underwent indirect radionuclide cystography. In total, 372 patients (55\%) underwent some form of imaging study for identifying VUR (-Table 1).

VUR was found in 125 of the 372 patients (34\%) who underwent VCUG. These included 64 patients (17\%) with dilating (grades 3-5) VUR (-Table 1). Of the 243 patients who underwent VCUG without an indication, VUR was found in 59 patients (24\%). These included 20 patients (8.2\%) with dilating VUR ( - Table 2). Patients with a NICE indication for VCUG were more likely have VUR $(p<0.0001)$ and highgrade VUR $(p<0.0001)$.

Since renal scans were performed only seldom (61 patients) in our department, and since the imaging modalities varied significantly, renal scans were excluded from this analysis. 
Table 2 Clinical consequences if the NICE guidelines had been stringently applied to the management of 672 children with UTI

\begin{tabular}{|c|c|c|c|c|c|}
\hline Age group & Boys $<6 \mathrm{mo}$ & Girls $<6 \mathrm{mo}$ & Boys $0.5-3$ y & Girls $0.5-3$ y & All patients \\
\hline US avoided in patients with normal US & 15 & 13 & 54 & 190 & 272 \\
\hline Abnormal US result missed & 4 & 0 & 8 & 32 & 44 \\
\hline Hydronephrosis missed & 0 & 0 & 1 & 2 & 3 \\
\hline Mild dilation missed & 2 & 0 & 2 & 14 & 18 \\
\hline Renal scarring missed & 0 & 0 & 2 & 13 & 15 \\
\hline Abnormal kidney size missed & 0 & 0 & 3 & 5 & 8 \\
\hline Duplicated ureter missed & 1 & 0 & 0 & 5 & 6 \\
\hline Bladder cyst missed & 0 & 0 & 1 & 1 & 2 \\
\hline Nephrocalcinosis missed & 1 & 0 & 0 & 0 & 1 \\
\hline Renal calculus missed & 0 & 0 & 0 & 1 & 1 \\
\hline Laterally positioned UVJ missed & 0 & 0 & 0 & 1 & 1 \\
\hline VCUG avoided in patients with no VUR & 65 & 30 & 18 & 71 & 184 \\
\hline VUR on VCUG missed & 16 & 8 & 10 & 25 & 59 \\
\hline Low-grade VUR missed & 13 & 5 & 4 & 17 & 39 \\
\hline High-grade VUR missed & 3 & 3 & 6 & 8 & 20 \\
\hline AMP for VUR not assigned & 15 & 7 & 8 & 19 & 49 \\
\hline Surgery not performed & 1 & 2 & 4 & 10 & 17 \\
\hline $\begin{array}{l}\text { Endoscopic injection treatment for } \\
\text { VUR not performed }\end{array}$ & 1 & 2 & 1 & 9 & 13 \\
\hline UNC not performed & 0 & 0 & 1 & 0 & 1 \\
\hline Nephrectomy not performed & 0 & 0 & 2 & 0 & 2 \\
\hline Pyeloplasty not performed & 0 & 0 & 0 & 1 & 1 \\
\hline
\end{tabular}

Abbreviations: AMP, antimicrobial prophylaxis; UNC, reteroneocystostomy; US, ultrasonography; UTI, urinary tract infection; UVJ, ureterovesical junction; VCUG, voiding cystourethrography; VUR, vesicoureteral reflux.

${ }^{a}$ Two patients underwent endoscopic injection treatment despite being diagnosed with only low-grade VUR.

\section{Antimicrobial Prophylaxis}

AMP was prescribed to 110 of the 125 patients with VUR, 49 of whom had undergone VCUG without an indication. AMP was, however, prescribed quite liberally, and 49 of the 61 patients with only low-grade VUR received AMP.

\section{Surgical Interventions}

A total of 49 patients underwent some form of urological surgery. Endoscopic injection treatment was performed on 30 patients, 13 of whom did not have an indication for VCUG. However, two of these patients had only low-grade VUR. Ureteroneocystostomy was performed on five patients, one of whom did not have an indication for any imaging studies. A nephrectomy was performed on two patients-both had abnormal RBUS findings and no indication for any imaging studies. Hydronephrosis in RBUS led to seven patients undergoing pelveoplasty for obstruction of the ureteropelvic junction. Of these patients, one had no indication for any imaging studies. Ureterocele was surgically treated in three patients, all of whom were diagnosed by RBUS for which they had an indication. A posterior urethral valve was endoscopically ablated after a RBUS showing dilation of the urinary tract led to further investigations. Both patients had a positive indication for RBUS. A nephrostomy was performed due to a renal empyema that was diagnosed by RBUS for which the patient had a positive indication. A circumcision was performed on one patient with the poor urine flow due to phimosis (-Tables 1 and 2).

\section{Discussion}

If the NICE guidelines for imaging studies had been stringently applied to this cohort of 672 children, VUR would have been missed in 59 patients, and this figure included no less than 20 of a total of 64 patients with high-grade VUR, that is, $31 \%$ of patients with dilating VUR would have been missed. In addition, 17 patients would not have undergone surgery.

Tse et $\mathrm{al}^{14}$ reported that in a cohort of 134 patients younger than 6 months, 25 refluxing ureters and 22 scarred kidneys would not have been found, if the NICE guidelines had been applied. Moreover, in accordance with our results, Lytzen et $\mathrm{al}^{16}$ stated that in a cohort of 96 patients, 5 of the 9 patients with VUR would have been missed, had the NICE guidelines been applied. In our previous article on the preliminary analyses of a subpopulation of this cohort, we reported that the NICE guidelines were adequate only in boys younger than 6 months. 
In support of the use of the NICE guidelines, Wong et $\mathrm{al}^{15}$ found in a large cohort of 820 patients, that the NICE guidelines had quite a high sensitivity, and 21 patients (2.6\%) with remediable urological abnormalities would have been missed. They also suggested revising the guidelines slightly, so as to make indications for imaging studies of boys less stringent. Jose and Sharif ${ }^{11}$ found that in a cohort of 48 patients aged 0 to 15 years, missing 1 patient with grade 3 VUR was the only significant consequence of applying the NICE guidelines. Schroeder et $\mathrm{al}^{13}$ stated that following the NICE guidelines in a prospective cohort resulted in decreased rates of VCUG and AMP, while detection rates of grades 4 to 5 VUR and of UTI recurrence were largely unaffected.

If the goal is to reduce the amount of unnecessary imaging studies, the diagnostic accuracy of UTI and determining the level of infection is of utmost importance. In the cohort examined in this article there were 130 patients who were erroneously diagnosed with $\mathrm{PN}$, although the diagnostic criteria were not met, and 37 patients who were diagnosed with cystitis, although the diagnostic criteria for PN were met.

Prenatal ultrasonography (US) screening for fetal anomalies is performed between 18 and 21 weeks of pregnancy both in the United Kingdom and in Finland. Although the detection of hydronephrosis in prenatal US significantly reduces the number of patients that are diagnosed with hydronephrosis for the first time after a UTI, there was a substantial number of patients (92 patients, 14\%) in our cohort with UTI and dilation in RBUS, who had previously undergone an antenatal US with normal results. We cannot, however, rule out the possibility that there might be differences in the sensitivity of detecting prenatal hydronephrosis by US between the United Kingdom and Finland. Naturally, the higher the quality is of prenatal US screening and the sensitivity for detecting prenatal hydronephrosis, the smaller the need is to perform RBUS after a UTI in infants and small children. There is, however, a small population of children, who develop hydronephrosis after birth, in whom there is a need for RBUS after a UTI, despite a normal prenatal US. RBUS is a well-tolerated imaging study that can be performed during initial hospitalization. Lee et $\mathrm{al}^{18}$ found RBUS results to be abnormal in $86 \%$ of patients with high-grade VUR. In our cohort, 51 of the 125 patients (41\%) with VUR, and 44 of the 64 patients (69\%) with highgrade VUR had an abnormal US after a UTI.

There is little evidence that recurrent UTIs or renal scarring in children lead to chronic kidney disease later in life in structurally healthy kidneys. ${ }^{19}$ Children with underlying anatomical abnormalities, however, are susceptible to these changes. ${ }^{4}$ Bearing this in mind, together with the fact that a large proportion $(21 \%)$ of children with normal antenatal RBUSs had an abnormal finding after a UTI, we strongly encourage performing RBUS routinely in all children with a first UTI.

If RBUS had been performed on all patients, and VCUG on all patients with abnormal RBUS findings, 12 of the 20 "missed" patients with dilating VUR would have been identified. Of the remaining eight patients, one patient had recurrent UTIs after endoscopic injection treatment, one had recurrent UTIs while taking AMP. Of the other six patients, five were diagnosed with a first UTI very early in life, before the age of 3 months. The remaining one patient was diagnosed with a first UTI (PN) at the age of 25 months and had no atypical features or recurrent UTIs. In other words, it is possible to determine those at risk for high-grade VUR quite well, but the dilemma remains: How many unnecessary imaging studies are justified to find one patient with highgrade VUR, and for whom is high-grade VUR a significant finding?

If the strategy of performing RBUS on all UTI patients, and VCUG on all patients with abnormal RBUS findings or atypical or recurrent UTIs (as defined by NICE) were applied in our cohort of 672 patients, all 272 patients who underwent a RBUS without a NICE indication and with normal RBUS findings would have undergone RBUS. Of the 243 patients who underwent VCUG without a NICE indication, 80 would have undergone VCUG. This would have resulted in 25 more patients with VUR, including 14 with dilating VUR, being identified; only 34 patients with VUR, including 6 with dilating VUR, would have been missed.

Recently, alternative imaging strategies have been proposed. One of these is the so-called "top-down" approach, whereby DMSA scan is the first-line imaging study after a UTI, and VCUG is performed only if the DMSA result is abnormal. Preda et $\mathrm{al}^{20}$ found that in a cohort of 290 patients, only 1 patient with dilating VUR would have been missed by taking this approach. In a study by Tseng et al, ${ }^{21}$ DMSA had a sensitivity of $88 \%$ for identifying patients with VUR, and $100 \%$ in identifying patients with high-grade VUR. In a cohort of 220 patients, combining US and DMSA results had a sensitivity of $95.3 \%$ in identifying patients with high-grade VUR. ${ }^{18}$ This strategy is supported by the rationale that VUR is most significant in patients who have other concurrent urological anomalies.

The effectiveness of AMP has been questioned. According to a prospective study AMP may reduce the rate of febrile UTI recurrences and new renal damage in girls, but boys benefit from neither treatment. ${ }^{22}$ Another review found that AMP has no significant effect on the incidence of febrile or symptomatic UTI, but may reduce the rate of new renal damage as assessed by DMSA scanning. ${ }^{23}$ As of yet, AMP has not conclusively been found to be ineffective. The problem may very well be one of compliance, owing to poor tolerance of antimicrobial drugs and unwillingness to commit to such a disciplined drug regimen in general.

The prospective study by Brandström et $\mathrm{al}^{22}$ showed that endoscopic injection treatment reduces the rate of febrile UTIs in girls, but not in boys. A meta-analysis of 5,527 patients ${ }^{24}$ arrived at an aggregate success rate of $85 \%$ of one or more injections. The rate of febrile UTI recurrence was $0.75 \%$.

Garin et $\mathrm{al}^{25}$ stated that patients with grades 1 to 3 VUR are not at greater risk of recurrent UTI, than those without VUR, and that AMP provides no statistically significant reduction in the rate of UTI recurrence or new renal scarring in patients with or without grades 1 to 3 VUR. Recently, consensus has shifted more toward the notion that low-grade VUR is indeed a benign condition that requires no treatment or follow-up. 
Some pediatric urologists consider even high-grade VUR to be inconsequential. ${ }^{26}$ This is supported by the findings of Moorthy et $\mathrm{al}^{27}$ and Gordon et al, ${ }^{28}$ according to whom VUR does not predict a higher risk for renal damage following UTI. The occurrence of VUR in urologically healthy children has not been conclusively evaluated since a historical survey 50 years ago, ${ }^{29}$ due to ethical issues regarding performing a VCUG in healthy children.

This is a retrospective study, and as such has certain caveats. The diagnostic reliability may be questionable in some cases. Suprapubic aspiration was frequently unsuccessfully attempted, leaving no other choice than to base the diagnosis on urinary bag specimens or clean voided samples, when possible. Blood work was inconsistent. Bacterial cultures of blood were seldom performed in infants in clinically good condition. Blood creatinine was also measured in only a part of patients. If blood work had been more uniform, more patients might have had a NICE indication for imaging. DMSA scintigraphy was performed on very few patients, and thus cannot be reliably assessed. Performing a DMSA scan to more patients might have spiked an interest in VCUG for more patients, as well. VCUG was performed in only 372 patients, 194 of whom underwent standard VCUG; thus the grading of VUR may not be entirely reliable in all cases.

Till this day, the optimal imaging algorithm for children with UTI remains unclear. A large prospective study is required to determine the most effective schedule for imaging. There is a need for further discussions on the pertinence of the NICE guidelines with regard to imaging studies in pediatric patients with UTI.

\section{Conclusion}

This study shows that the NICE guidelines cannot, as such, be recommended for imaging studies in children younger than 3 years with UTI. If the NICE guidelines had been stringently applied in our cohort of 672 patients, VUR would have been missed in 59 patients (47\%), including 20 (31\%) with highgrade VUR and 17 patients would not have undergone surgery that was considered necessary for the maintenance of good health into adulthood.

\section{Conflict of Interest}

None.

\section{References}

1 Shaikh N, Morone NE, Bost JE, Farrell MH. Prevalence of urinary tract infection in childhood: a meta-analysis. Pediatr Infect Dis J 2008;27(4):302-308

2 Hansson S, Martinell J, Stokland E, Jodal U. The natural history of bacteriuria in childhood. Infect Dis Clin North Am 1997;11(3): 499-512

3 Shaikh N, Ewing AL, Bhatnagar S, Hoberman A. Risk of renal scarring in children with a first urinary tract infection: a systematic review. Pediatrics 2010;126(6):1084-1091

4 Swerkersson S, Jodal U, Sixt R, Stokland E, Hansson S. Relationship among vesicoureteral reflux, urinary tract infection and renal damage in children. J Urol 2007;178(2):647-651, discussion 650-651

5 National Institute for Health and Clinical Excellence. NICE clinical guideline 54. Urinary tract infection in children: Diagnosis, treatment and long-term management. Available at: http://www.nice.org.uk/ nicemedia/live/11819/36032/36032.pdf Accessed December 12, 2013

6 Roberts KB; Subcommittee on Urinary Tract Infection, Steering Committee on Quality Improvement and Management. Urinary tract infection: clinical practice guideline for the diagnosis and management of the initial UTI in febrile infants and children 2 to 24 months. Pediatrics 2011;128(3):595-610

7 Jodal U, Lindberg U; Swedish Medical Research Council. Guidelines for management of children with urinary tract infection and vesico-ureteric reflux. Recommendations from a Swedish stateof-the-art conference. Acta Paediatr Suppl 1999;88(431):87-89

8 Riccabona M, Avni FE, Blickman JG, et al. Imaging recommendations in paediatric uroradiology: minutes of the ESPR workgroup session on urinary tract infection, fetal hydronephrosis, urinary tract ultrasonography and voiding cystourethrography, Barcelona, Spain, June 2007. Pediatr Radiol 2008;38(2):138-145

9 Ammenti A, Cataldi L, Chimenz R, et al; Italian Society of Pediatric Nephrology. Febrile urinary tract infections in young children: recommendations for the diagnosis, treatment and follow-up. Acta Paediatr 2012;101(5):451-457

10 European Association of Urology. Guidelines: Online Guidelines (2012). Available at: http://www.uroweb.org/guidelines/onlineguidelines/. Accessed December 12, 2013

11 Jose SS, Sharif ES. New NICE guidelines for UTI management: what will change? Arch Dis Child 2008;93(8):716-717

12 Ristola MT, Hurme T. NICE guidelines for imaging studies in children with UTI adequate only in boys under the age of 6 months. Pediatr Surg Int 2013;29(3):215-222

13 Schroeder AR, Abidari JM, Kirpekar R, et al. Impact of a more restrictive approach to urinary tract imaging after febrile urinary tract infection. Arch Pediatr Adolesc Med 2011;165(11): 1027-1032

14 Tse NK, Yuen SL, Chiu MC, Lai WM, Tong PC. Imaging studies for first urinary tract infection in infants less than 6 months old: can they be more selective? Pediatr Nephrol 2009;24(9): 1699-1703

15 Wong SN, Tse NK, Lee KP, et al. Evaluating different imaging strategies in children after first febrile urinary tract infection. Pediatr Nephrol 2010;25(10):2083-2091

16 Lytzen R, Thorup J, Cortes D. Experience with the NICE guidelines for imaging studies in children with first pyelonephritis. Eur J Pediatr Surg 2011;21(5):283-286

17 Lebowitz RL, Olbing H, Parkkulainen KV, Smellie JM, TamminenMöbius TE; International Reflux Study in Children. International system of radiographic grading of vesicoureteric reflux. Pediatr Radiol 1985;15(2):105-109

18 Lee HY, Soh BH, Hong CH, Kim MJ, Han SW. The efficacy of ultrasound and dimercaptosuccinic acid scan in predicting vesicoureteral reflux in children below the age of 2 years with their first febrile urinary tract infection. Pediatr Nephrol 2009;24(10): 2009-2013

19 Salo J, Ikäheimo R, Tapiainen T, Uhari M. Childhood urinary tract infections as a cause of chronic kidney disease. Pediatrics 2011; 128(5):840-847

20 Preda I, Jodal U, Sixt R, Stokland E, Hansson S. Normal dimercaptosuccinic acid scintigraphy makes voiding cystourethrography unnecessary after urinary tract infection. J Pediatr 2007;151(6): 581-584, e1

21 Tseng MH, Lin WJ, Lo WT, Wang SR, Chu ML, Wang CC. Does a normal DMSA obviate the performance of voiding cystourethrography in evaluation of young children after their first urinary tract infection? J Pediatr 2007;150(1):96-99 
22 Brandström P, Jodal U, Sillén U, Hansson S. The Swedish reflux trial: review of a randomized, controlled trial in children with dilating vesicoureteral reflux. J Pediatr Urol 2011;7(6):594-600

23 Nagler EV, Williams G, Hodson EM, Craig JC. Interventions for primary vesicoureteric reflux. Cochrane Database Syst Rev 2011; (6):CD001532

24 Elder JS, Diaz M, Caldamone AA, et al. Endoscopic therapy for vesicoureteral reflux: a meta-analysis. I. Reflux resolution and urinary tract infection. J Urol 2006;175(2):716-722

25 Garin EH, Olavarria F, Garcia Nieto V, Valenciano B, Campos A, Young L. Clinical significance of primary vesicoureteral reflux and urinary antibiotic prophylaxis after acute pyelonephritis: a multicenter, randomized, controlled study. Pediatrics 2006;117(3):626-632
26 Venhola M, Uhari M. Vesicoureteral reflux, a benign condition. Pediatr Nephrol 2009;24(2):223-226

27 Moorthy I, Easty M, McHugh K, Ridout D, Biassoni L, Gordon I. The presence of vesicoureteric reflux does not identify a population at risk for renal scarring following a first urinary tract infection. Arch Dis Child 2005;90(7):733-736

28 Gordon I, Barkovics M, Pindoria S, Cole TJ, Woolf AS. Primary vesicoureteric reflux as a predictor of renal damage in children hospitalized with urinary tract infection: a systematic review and meta-analysis. J Am Soc Nephrol 2003;14(3):739-744

29 Köllermann MW, Ludwig H. On vesico-ureteral reflux in normal infants and children [in German]. Z Kinderheilkd 1967;100(3): 185-191 